\title{
MicroRNA-138 inhibits proliferation, migration and invasion through targeting hTERT in cervical cancer
}

\author{
NA ZHOU ${ }^{1}$, DAN FEI ${ }^{2}$, SHAN ZONG $^{1}$, MINGYUE ZHANG $^{1}$ and YING YUE ${ }^{1}$ \\ ${ }^{1}$ Department of Obstetrics and Gynecology, The First Hospital of Jilin University, Changchun, Jilin 130021; \\ ${ }^{2}$ Department of Ultrasonography, China-Japan Union Hospital of Jilin University, Changchun, Jilin 130033, P.R. China
}

Received April 25, 2015; Accepted June 29, 2016

DOI: $10.3892 / \mathrm{ol} .2016 .5038$

\begin{abstract}
A growing body of evidence suggests that microRNA-138 (miR-138) functions as a tumor suppressor, and is involved in tumor initiation, development and metastasis in certain types of human cancers. However, the function and underlying molecular mechanism of miR-138 in cervical cancer remains unclear. Therefore, the purpose of the present study was to investigate the clinical significance of miR-138 expression in cervical cancer, and to evaluate its role and underlying mechanisms in cervical cancer. The present study indicated that miR-138 expression was significantly downregulated in cervical cancer tissues and cell lines, and that the low miR-138 expression was negatively associated with advanced FIGO stage and lymph node metastasis $(\mathrm{P}<0.01)$. Functional analyses indicated that the overexpression of miR-138 in cervical cancer cells inhibited cell proliferation, migration and invasion, induced cell apoptosis in vitro, and suppressed tumor growth in a nude mice model. Luciferase reporter assays confirmed that human telomerase reverse transcriptase was a novel target gene of miR-138. The findings of the present study suggested that miR-138 could be a potential candidate for cervical cancer therapeutics.
\end{abstract}

\section{Introduction}

Cervical cancer is the third most common cancer in women globally, with an estimated global incidence of $>500,000$ novel cases and a tremendously high number of mortalities ( 274,000$)$, annually (1). Although radiotherapy, chemotherapy and surgery have been recently used as standard treatment to improve overall survival, progression free survival and recurrence rates for patients with

Correspondence to: Professor Ying Yue, Department of Obstetrics and Gynecology, The First Hospital of Jilin University, 71 Xinmin Street, Chaoyang, Changchun, Jilin 130021, P.R. China

E-mail: yueying2514@sina.com

Key words: cervical cancer, human telomerase reverse transcriptase, microRNAs, microRNA-138 cervical cancer $(2,3)$, the 5-year survival rates for advance stage cervical cancer (stage III and IV patients) remains at $<40 \%(4,5)$. In addition, $\sim 30 \%$ of patients with this disease experience lymph node recurrence and distant metastasis after primary treatment (6). Therefore, uncovering the molecular mechanisms responsible for the development of cervical cancer is crucial for identifying potential therapeutic targets for cervical cancer.

MicroRNAs (miRNAs/miRs) are non-coding RNA molecules of 19-25 nucleotides in length that modulate the translational efficiency or stability of target messenger RNAs (mRNAs), predominantly through targeting of the 3' untranslated regions (3'UTRs) of mRNAs (7). Over the past decade, emerging evidences have indicated that miRNAs are critical regulators of cancer occurrence and progression that are involved in cancer cell proliferation, differentiation, apoptosis and metastasis $(8,9)$. miRNAs are abnormally expressed in human cancers and can act as either oncogenes by repressing tumor suppressors or tumor suppressors by negatively regulating oncogenes (10). In recent years, miRNAs have been used as novel targets for anticancer therapies and as molecular diagnosis marker or prognostic markers in various cancers, including cervical cancer $(11,12)$.

There is particularly a growing interest toward miR-138, in the context of numerous cancers. miR-138, a family of microRNA precursors, has been reported to function as a tumor suppressor in a variety of human cancers, including renal carcinoma (13), non-small lung cancer (14), colorectal cancer (15), neuroblastoma (16), esophageal squamous cell carcinoma (17), nasopharyngeal carcinoma (18) and hepatocellular carcinoma (19). However, the role and the molecular mechanisms of miR-138 in cervical remain unclear.

Therefore, the aim of the present study was to investigate the clinical significance of miR-138 expression in cervical cancer, and to evaluate its role and underlying mechanisms in cervical cancer. The results of the study showed that miR-138 expression was downregulated in cervical cancer tissues and cell lines. Overexpression of miR-138 in cervical cancer cells inhibited cell growth in vitro and tumor growth in nude mice. Furthermore, the present study showed that human telomerase reverse transcriptase (hTERT) was a direct target of miR-138 in cervical cancer. These findings provide a novel therapeutic strategy for the treatment of cervical cancer. 


\section{Materials and methods}

Ethics statement. Written informed consent was obtained from patients according to a protocol approved by the Ethics Committee of the First of Hospital of Jilin University (Changchun, China). Animal experiments were performed in strict accordance with the protocols approved by the Animal Care Committee of the Jilin University.

Patients and tissue samples. In total, 36 pairs of cervical cancer samples and matched normal cervical tissues were collected from 36 patients, who underwent surgery between October 2010 and July 2014 at the First Hospital of Jilin University (Changchun, China). The specimens were collected immediately after surgery and stored in liquid nitrogen prior to further use. The category of cervical samples was confirmed by pathological analysis, as previously described (20). The patient clinical information was listed in Table I.

Cell lines and transfections. HaCaT cells (an immortalized HPV-negative skin keratinocyteline) and two cervical cancer cell lines, HeLa and SiHa cells, were obtained from the Type Culture Collection of the Chinese Academy of Sciences (Shanghai, China), and were maintained in Dulbecco's modified Eagle's medium (DMEM; Invitrogen; Thermo Fisher Scientific, Inc., Waltham, MA, USA) containing $10 \%$ fetal bovine serum (FBS; HyClone; GE Healthcare Life Sciences, Logan, UT, USA). All cells were cultured at $37^{\circ} \mathrm{C}$ in a humidified atmosphere containing $5 \% \mathrm{CO}_{2}$.

miR-138 mimic and a corresponding negative control miRNA (miR-NC) were obtained from Shanghai GenePharma Co., Ltd., (Shanghai, China), and were transiently transfected into HeLa cells at a concentration of $50 \mathrm{nM}$ using Lipofectamine 2000 (Invitrogen; Thermo Fisher Scientific, Inc.), according to the manufacturer's instructions.

Quantitative reverse transcription-polymerase chain reaction ( $q R T-P C R$ ). Total RNA from cells ( $\mathrm{SiHa}, \mathrm{HaCaT}$ and HeLa) or tissue was isolated using Trizol reagent (Invitrogen; Thermo Fisher Scientific, Inc.) according to the manufacturer's instructions. Total RNA was reverse-transcribed using the Takara PrimeScript ${ }^{\mathrm{TM}}$ First Strand cDNA Synthesis kit (Takara Bio, Inc., Otsu, Japan) according to the manufacturer's instructions. The levels of miR-138 were determined using a TaqMan MicroRNA Assays kit (TaqMan assay ID, 002284; measuring mature miR-138; Applied Biosystems; Thermo Fisher Scientific Inc.) as previously described (21), on an ABI Applied Biosystems 7900 Real Time PCR system (Applied Biosystems; Thermo Fisher Scientific, Inc.). The U6 small RNA was used as an internal control. The primers used were as follows: miR-138 forward, 5'-AGCTGGTGT TGTGAATCAGGCCG-3' and reverse, 5'-TGGTGTCGT GGAGTCG-3'; and U6 forward, 5'-CTCGCTTCGGCAGCA CA-3' and reverse, 5'-AACGCTTCACGAATTTGCGT-3'. The PCR cycling conditions were $95^{\circ} \mathrm{C}$ for $10 \mathrm{~min}$, and 40 cycles of $15 \mathrm{sec}$ at $95^{\circ} \mathrm{C}$ and $30 \mathrm{sec}$ at $60^{\circ} \mathrm{C}$, followed by an annealing/elongation step at $72^{\circ} \mathrm{C}$ for $5 \mathrm{~min}$. The comparative $2^{-\Delta \Delta C q}$ method (22) was used for relative quantification and statistical analysis.
Table I. Association between miR-138 expression and the clinicopathological features of human cervical cancer.

\begin{tabular}{lccc}
\hline Feature & $\begin{array}{c}\text { No. of } \\
\text { patients }\end{array}$ & $\begin{array}{c}\text { Relative } \\
\text { miR-138 level }\end{array}$ & P-value \\
\hline $\begin{array}{l}\text { Age, years } \\
\quad 55\end{array}$ & 16 & $0.42 \pm 0.11$ & 0.743 \\
$\quad 255$ & 20 & $0.42 \pm 0.09$ & \\
$\begin{array}{l}\text { Tumor size } \\
\quad 55 \mathrm{~cm}\end{array}$ & 19 & $0.41 \pm 0.10$ & 0.718 \\
$\quad 5 \mathrm{~cm}$ & 17 & $0.43 \pm 0.08$ & \\
$\begin{array}{l}\text { Histological grades } \\
\text { Well/moderate }\end{array}$ & 24 & $0.44 \pm 0.12$ & \\
$\quad$ Poor & 12 & $0.38 \pm 0.09$ & \\
FIGO stage & & & $<0.010$ \\
$\quad$ Ib-IIa & 22 & $0.53 \pm 0.11$ & \\
$\quad$ IIb-IIIa & 14 & $0.25 \pm 0.06$ & \\
Lymph node & & & $<0.010$ \\
metastasis & & & \\
$\quad$ No & 28 & $0.48 \pm 0.08$ & \\
Yes & 8 & $0.21 \pm 0.06$ & \\
\hline
\end{tabular}

miR-138, microRNA-138; FIGO, International Federation of Gynecology and Obstetrics.

Assessment of cell viability and proliferative capacity. To determine the effect of miR-138 on cell viability and proliferative capacity in cervical cancer cells, 3-(4,5-dimethylthiazol-2-yl)-2,5-diphenyltetrazolium bromide (MTT) and colony formation assays were performed in HeLa cells following transfection with miR-138 mimic or miR-NC. For cell viability, $5 \times 10^{3}$ cells were seeded into 96 -well plates with $100 \mu$ l of DMEM and incubated for $24 \mathrm{~h}$. Cells were then transfected with miR-138 or miR-NC, and cultured for 24,48 or $72 \mathrm{~h}$. Subsequently, $20 \mu \mathrm{l}$ MTT ( $5 \mathrm{mg} / \mathrm{ml}$; Sigma-Aldrich, St. Louis, MO, USA) was added to the samples, and cultured for $4 \mathrm{~h}$ at $37^{\circ} \mathrm{C}$. Following incubation, $200 \mu$ l of dimethyl sulfoxide (DMSO; Sigma-Aldrich) was added to solubilize the crystals for $20 \mathrm{~min}$ at room temperature. Cell viability was assessed at an absorbance of $490 \mathrm{~nm}$ using a biorad iMark Microplate Absorbance Reader (BD Biosciences, Franklin Lakes, NJ, USA).

For the colony formation assay, the number of viable HeLa cell colonies was determined after 14 days, following the inoculation of 1,000 cells/well in triplicate in 6-well plates. The cells were fixed with 4\% paraformaldehyde (Sigma-Aldrich), and stained with $1 \%$ crystal violet (Sigma-Aldrich). The percentage colony formation was calculated by adjusting control cells to $100 \%$.

Cell apoptosis assay. The percentage of apoptotic cells was assessed by flow cytometry. In brief, HeLa cells were transfected with miR-138 mimic or miR-NC for $48 \mathrm{~h}$, and then apoptotic cells were determined using the AnnexinV/propidium iodide detection kit (Nanjing KeyGen Biotech. Co. Ltd., Nanjing, China), according to the manufacturer's instructions. The apoptotic rate were measured by using a FACS Calibur 
flow cytometer (BD Biosciences), and the data were analyzed using CellQuest software (version 5.1; BD Biosciences).

Cell migration and invasion assays. For the Transwell migration assay, $1 \times 10^{5}$ transfected HeLa cells in $200 \mu \mathrm{l}$ of serum-free DMEM were seeded into the upper part of each Transwell chamber (pore size, $8-\mu \mathrm{m}$; Costar; Corning Incorporated, Corning, NY, USA) without Matrigel. For the invasion assay, $1 \times 10^{5}$ transfected cells were placed on the upper chamber of each insert coated with Matrigel (BD Biosciences). Subsequently, $500 \mu 1 \mathrm{DMEM}$ with $20 \%$ FBS was added to the lower part of the chamber. After incubating for $24 \mathrm{~h}$ at $37^{\circ} \mathrm{C}$ with $5 \%$ $\mathrm{CO}_{2}$, cells that had migrated or invaded to the lower surface of the filter were fixed in $70 \%$ ethanol (Sigma-Aldrich) for $30 \mathrm{~min}$ and stained with $2 \%$ crystal violet for $10 \mathrm{~min}$ on a glass slide. The invaded or migrated cells were photographed under an IX51 inverted microscope (Olympus Corporation, Tokyo, Japan) and counted in 5 randomly selected fields.

Plasmid construction and luciferase reporter assay. The 3'UTR of hTERT containing the putative miR-138 binding site (wild-type) was cloned into the pGL3-control vector (Ambion; Thermo Fisher Scientific, Inc.). A mutant 3'UTR of phosphatase and tensin homolog (mutant type) was synthesized by PCR and cloned into the pGL3-control vector. Subsequently, $5 \times 10^{3} \mathrm{HeLa}$ cells were seeded in a 24 -well plate and transiently co-transfected with wild-hTERT-UTR-pGL3 or mutant-hTERT-UTR-pGL3, a Renilla luciferase control vector (20 ng) and miR-138 mimic or miR-NC. Luciferase activity was measured using the Dual Luciferase Reporter Assay System (Promega Corporation, Madison, WI, USA) at $48 \mathrm{~h}$ post-transfection. The specific activity is expressed as the fold changes of the experimental group vs. the miR-NC group.

Western blot analysis. Tissue or HeLa cell samples were harvested and lysed in ice-cold radioimmunoprecipitation assay buffer (Santa Cruz Biotechnology, Inc., Dallas, TX, USA) to extract proteins, according to the manufacturer's instructions. The concentrations of the final protein samples were determined with a bicinchoninic acid protein assay kit (Pierce Biotechnology, Inc., Rockford, IL, USA) Protein sample (30 $\mu \mathrm{g}$ each lane) was separated on $10 \%$ sodium dodecyl sulfate-polyacrylamide gel electrophoresis and electroblotted to a polyvinylidene difluoride membrane (EMD Millipore, Billerica, MA, USA). Membranes were blocked with $5 \%$ non-fat dried milk in $1 \mathrm{X}$ Tris-buffered saline containing $0.1 \%$ Tween 20 (TBST; Sigma-Aldrich) buffer for $2 \mathrm{~h}$ and then incubated with primary antibodies separately overnight at $4^{\circ} \mathrm{C}$. The primary antibodies included mouse monoclonal anti-human $\beta$-actin (dilution, 1:5,000; catalog no., sc-47778; Santa Cruz Biotechnology, Inc.) and rabbit polyclonal anti-human hTERT (dilution, 1:1,000; catalog no., sc-7212; Santa Cruz Biotechnology, Inc.). $\beta$-actin was used as the internal control. Membranes were washed with TBST three times and then further incubated with horseradish peroxidase (HRP)-conjugated polyclonal goat anti-rabbit immunoglobulin G (dilution, 1:5,000; catalog no., sc-2004; Santa Cruz Biotechnology, Inc.) or HRP-conjugated polyclonal goat-anti-mouse IgG (1:5,000; catalog no. sc-2005; Santa Cruz Biotechnology) for $2 \mathrm{~h}$ at room temperature. Signal intensities were detected using the Odyssey Infrared Imaging System (Li-COR Biosciences, Lincoln, NE, USA) using Odyssey v1.2 software (Li-COR Biosciences).

Xenograft experiments. Five week-old female BALB/c nu/nu mice (18-20 g) were purchased from Jilin Institute of Experimental Animals (Changchun, China), and bred at the Animal Laboratory Center, Jilin University (Changchun, China).

Equal numbers of HeLa cells $\left(2 \times 10^{6}\right)$ with forced expression of miR-138 mimic or scramble were suspended in $100 \mu \mathrm{l}$ serum-free DMEM and injected subcutaneously into the right rear flank of each mouse $(n=10)$. Tumor volume was periodically blindly measured by caliper every 5 days until mice were sacrificed under anesthesia. The formula for tumor volume was as follows: $\mathrm{V}$ (volume) $=1 / 2 \times \mathrm{A} \mathrm{x} \mathrm{B}^{2}$, where $\mathrm{A}$ and $\mathrm{B}$ are the longest and shortest diameters, respectively. The mice were sacrificed 25 days subsequent to injection. The tumor tissues were dissected and weighed. A part of tumor tissues was used to measure the hTERT level by western blot analysis, using the aforementioned method.

Statistical analysis. Experimental data are presented as the mean \pm standard deviation based on the results of at least 3 repeats. Statistical analysis between two samples was performed using a two-tailed Student's $t$-test, and analysis between more than two groups was performed using one-way analysis of variance, followed by Tukey's post hoc test. GraphPad Prism 5.0 software (GraphPad Software, Inc., La Jolla, CA, USA) was used for statistical analyses. A value of $\mathrm{P}<0.05$ was considered to indicate a statistically significant difference.

\section{Results}

miR-138 is downregulated in human cervical cancer cell lines and tissue specimens. A panel of human cervical cancer cell lines was first analyzed to quantitate the expression level of miR-138. The result showed that the expression level of miR-138 was downregulated in cervical cell lines compared with the normal cervical HaCaT cells (Fig. 1A). Additionally, the expression level of miR-138 in the HeLa cell line was decreased compared with the SiHa cell line; thus, HeLa cells were selected for the rest of the study.

The expression levels of miR-138 were examined in the 36 cervical cancer specimens and the patient-matched normal specimens. RT-PCR analysis showed that the expression of miR-138 was downregulated in $83.3 \%$ of the cervical tissues compared with the adjacent normal tissues; the average magnitude of this decrease was 2.1-fold (Fig. 1B).

In addition, the association between miR-138 expression and the clinical factors was assessed. As shown in Table I, the aberrant expression of miR-138 was associated with lymph node metastasis $(\mathrm{P}<0.01)$ and International Federation of Gynecology and Obstetrics $(\mathrm{FIGO})$ stage $(\mathrm{P}<0.01)$. There was no association between miR-138 expression and age, histological grade or tumor size. These findings suggest that miR-138 could be involved in the metastasis and progression of cervical cancer.

Overexpression of miR-138 inhibits cell viability and colony formation, but induces apoptosis in cervical cancer cells. To investigate the biological effects of miR-138 in cervical 
A

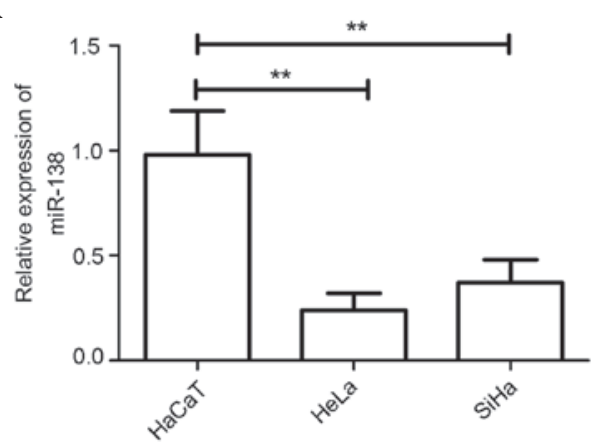

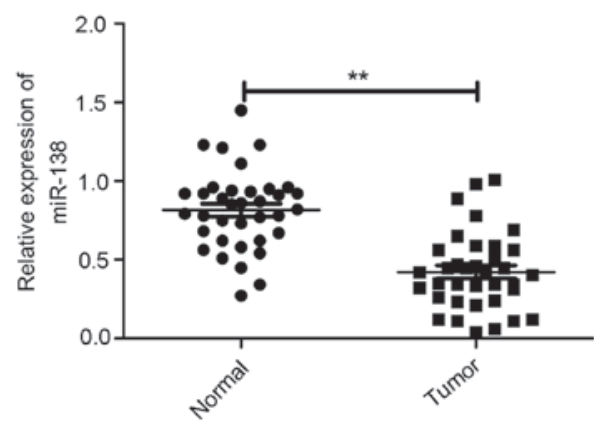

Figure 1. miR-138 expression was regulated in cervical cancer cell lines and tissues. (A) miR-138 was detected in two types of cervical cancer cells, and normal cervical squamous HaCaT cells by qRT-PCR. U6 was used as loading control. (B) miR-138 expression was detected in 36 pairs of cervical cancer tissue samples and adjacent normal tissue samples by qRT-PCR. ${ }^{* *} \mathrm{P}<0.01$ vs. HaCaT or normal control. miR-138, microRNA-138; qRT-PCR, quantitative reverse transcription-polymerase chain reaction; Normal, adjacent normal tissue; Tumor, cervical cancer tissue.

A

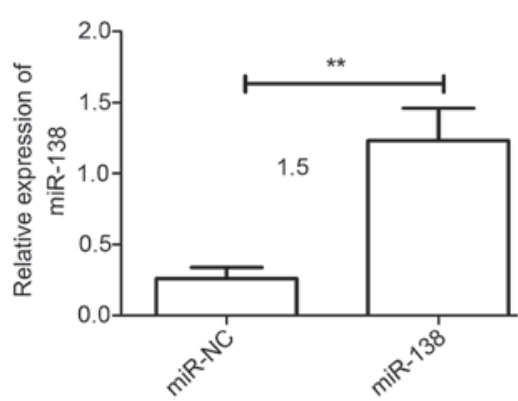

C

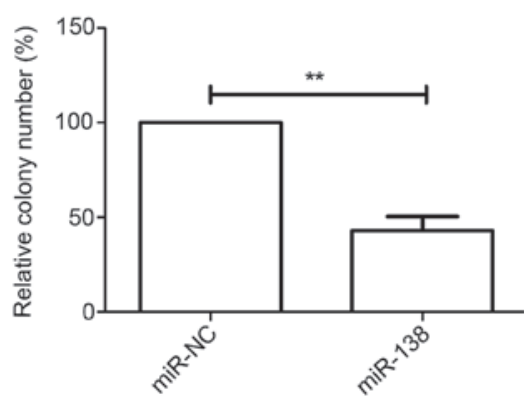

B

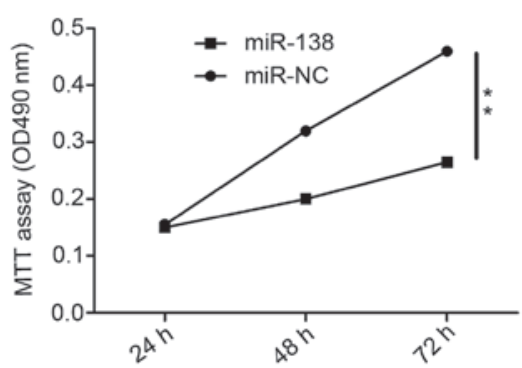

D

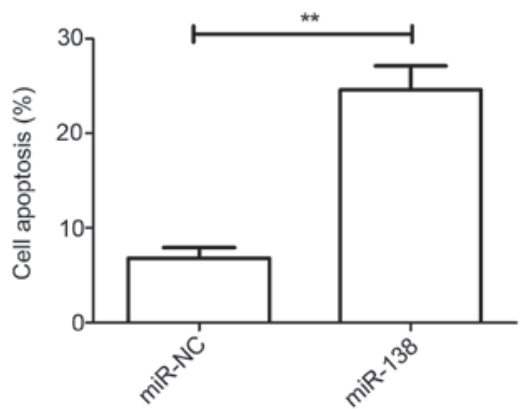

Figure 2. Overexpression of miR-138 inhibits proliferation and colony formation, and induces apoptosis of cervical cancer cells. (A) Expression of miR-138 was detected in HeLa cells transfected with miR-138 mimic or miR-NC by quantitative reverse transcription-polymerase chain reaction. (B) Cell proliferation was determined by MTT assay in HeLa cells after being transfected with miR-138 mimic or miR-NC. (C) Colony formation was measured in HeLa cells after being transfected with miR-138 mimic or miR-NC. (D) Apoptosis was detected in HeLa cells after being transfected with miR-138 mimic or miR-NC. ${ }^{* *} \mathrm{P}<0.01$ vs. miR-NC. miR-138, microRNA-138; miR-NC, miR-negative control; MTT, 3-(4,5-dimethylthiazol-2-yl)-2,5-diphenyltetrazolium bromide; OD490, optical density at wavelength 490 .

cancer cells, the cervical cancer HeLa cell line was transfected with miR-138 mimic and then the function of miR-138 in the cervical cancer cells was evaluated. qRT-PCR analysis confirmed that the transfected miR-138 mimic resulted in the upregulation of miR-138 expression in cervical cancer cells compared with cells transfected with miR-NC (Fig. 2A). The MTT assay showed that transfection with miR-138 mimic inhibited cell proliferation compared with transfection with miR-NC (Fig. 2B). The colony formation assay demonstrated that the overexpression of miR-138 inhibited colony formation in cervical cancer cells (Fig. 2C). Flow cytometry as then used to test the role of miR-138 in apoptosis. The results showed that upregulated miR-138 induced apoptosis (Fig. 2D). These results suggest that miR-138 may function as a tumor suppressor in cervical cancer cells.
Overexpression of miR-138 inhibits cell migration and invasion in cervical cancer cells. The present study has showed that miR-138 expression is associated with lymph node metastasis in patients with cervical cancer. However, this finding does not determine whether miR-138 effects the migration and invasion of cervical cancer cells. Migration and invasion assays in cervical cancer cells were performed using Transwell chambers, after HeLa cells were transfected with miR-138 mimic or miR-NC. Consistent with the clinical data, the overexpression of miR-138 significantly decreased the migration and invasion capacities of HeLa cells ( $\mathrm{P}<0.05$; Fig. $3 \mathrm{~A}$ and $\mathrm{B})$.

hTERT is a direct target of miR-138. hTERT has been reported to be an important molecule for promoting cell proliferation and invasion in various cancers, including cervical 
A
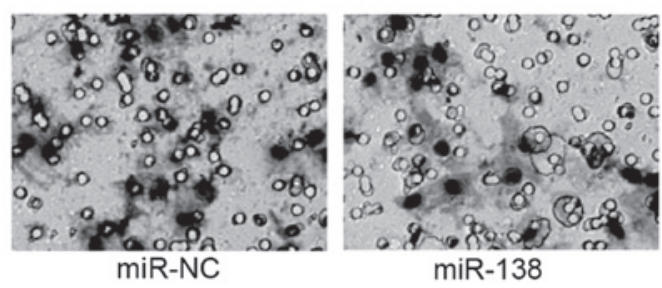

B

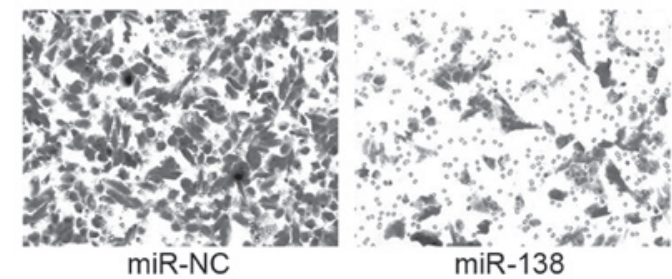

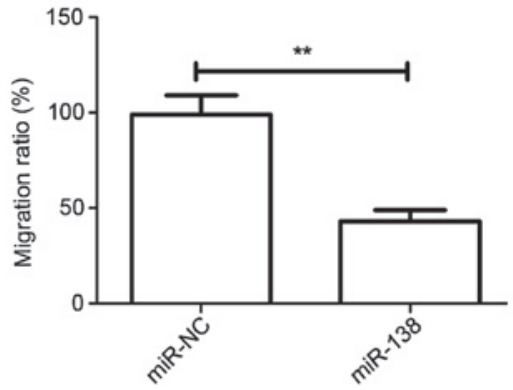

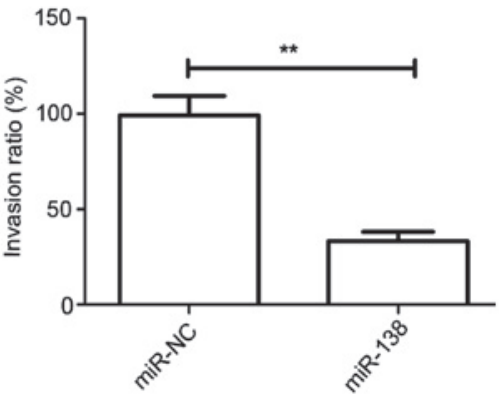

Figure 3. Overexpression of miR-138 inhibits the migration and invasion of cervical cancer cells. (A) Cell migration was determined using Transwell assays in HeLa cells that had been transfected with miR-138 mimic or miR-NC (200x). (B) Cell invasion was determined using a matrix penetration assay with Matrigel in HeLa cells that had been transfected with miR-138 mimic or miR-NC (100x). ${ }^{* *} \mathrm{P}<0.01$ vs. miR-NC. miR-138, microRNA-138; miR-NC, miR-negative control.

A

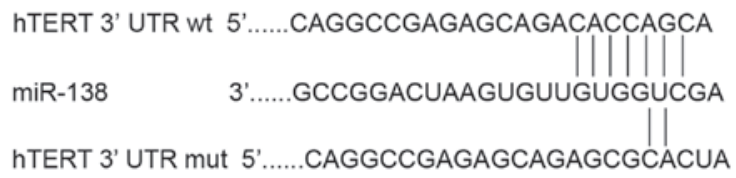

C

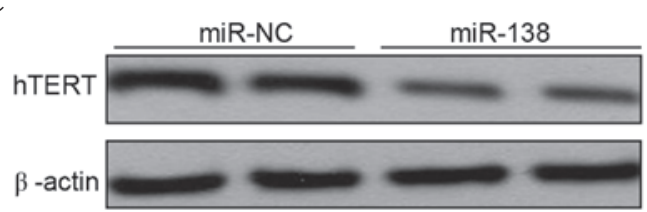

Figure 4. hTERT are direct targets of miR-138. (A) Predicted binding sites for miR-138 in the 3'UTR of hTERT and the mutations in the binding sites are shown. (B) Luciferase assay was performed to confirm the direct regulation of miR-138 on hTERT 3'UTR. ** $P<0.01$ vs. miR-NC. (C) hTERT protein expression in HeLa cells transfected with miR-138 mimic were detected by western blot analysis. $\beta$-actin was used as an internal control. hTERT, human telomerase reverse transcriptase; miR-138, microRNA-138; 3'UTR, 3' untranslated region; wt, wild type; mut, mutant type.

cancer (23). Using predictive tools (TargetScanHuman 7.1; www.targetscan.org/vert_71/), hTERT was hypothesized to be a target of miR-138 (Fig. 4A). To additionally confirm whether miR-138 directly targets the hTERT oncogene, luciferase reporter assays were performed. The luciferase assay showed that HeLa cells transfected with miR-138 significantly decreased wild-type hTERT-3'UTR reporter activity compared with the cells co-transfected with miR-NC $(\mathrm{P}<0.01)$, while miR-138 had no inhibitory effect on mutant hTERT-3'UTR reporter activity (Fig. 4B), indicting the direct regulation of miR-138 in the 3'UTR of hTERT mRNA. To further validate the association between miR-138 and hTERT, endogenous hTERT protein expression
B

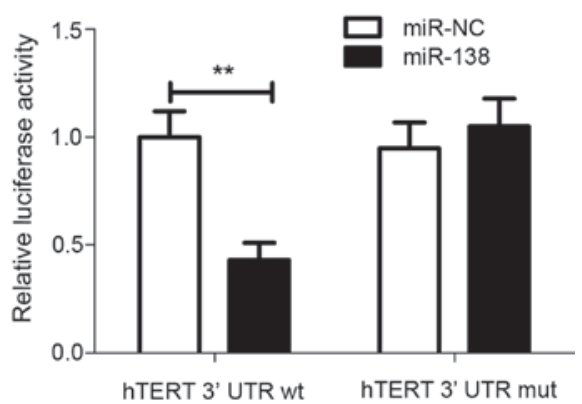


A

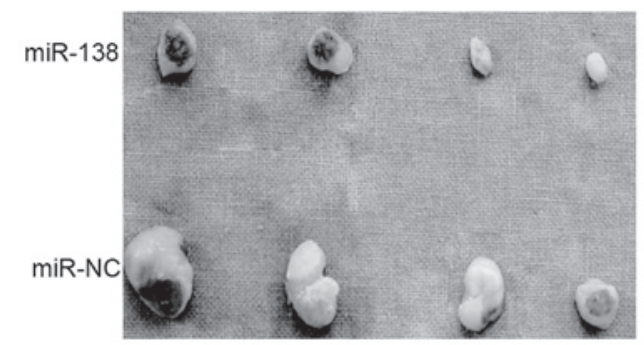

C

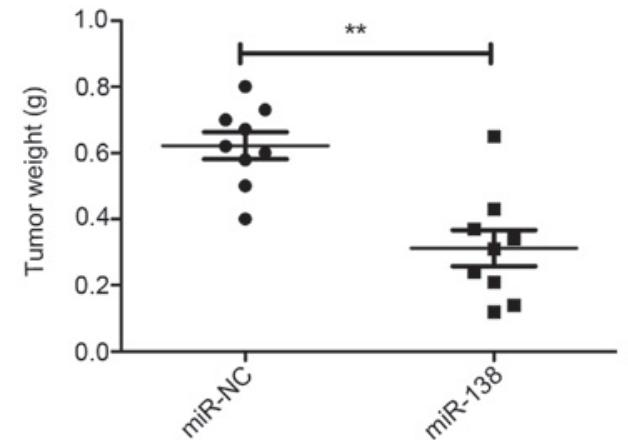

B

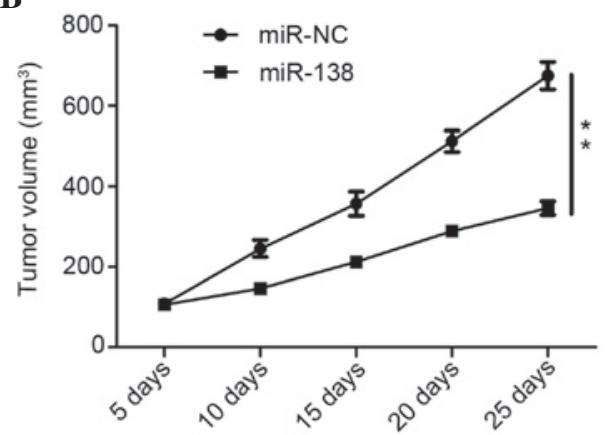

D

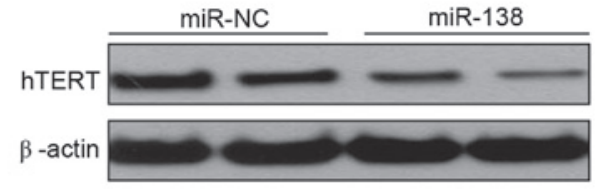

Figure 5. Overexpression of miR-138 suppressed tumor growth in HeLa xenografts. (A) Morphological characteristics of tumors in mice inoculated with HeLa/miR-138 or HeLa/miR-NC are shown. (B) Growth curves of tumor volumes in xenografts of nude mice. (C) Weight of tumor tissues was measured in each group. (D) hTERT was determined by western blot analysis. $\beta$-actin was used as an internal control. ${ }^{* *} \mathrm{P}<0.01$ vs. miR-NC group. miR-138, microRNA-138; miR-NC, miR-negative control; hTERT, human telomerase reverse transcriptase.

in tumor size (Fig. 5B) and weight (Fig. 5C) was observed in mice from the group injected with HeLa/miR-138 compared with the mice injected with HeLa/miR-NC (Fig. 5B and C). Furthermore, hTERT expression was also determined in tumor tissue using western blot analysis. Western blot analysis demonstrated that the overexpression of miR-138 significantly decreased hTERT expression. These findings suggest that miR-138 could suppress the growth of cervical cancer tumors in vivo by targeting hTERT.

\section{Discussion}

Discovering the molecules involved in cervical cancer cell initiation and progression and understanding the associated mechanisms are critical for developing effective therapeutic strategies to improve the survival and prognosis of patients suffering from cervical cancer. Growing evidence has demonstrated that numbers of miRNAs are crucial for the initiation, progression and metastasis of cervical cancer by regulating various processes, including cancer cell proliferation, differentiation, apoptosis, adhesion, cell cycle arrest, migration and invasion (11,24,25). For instance, Zhou et al (26) demonstrated that that miR-107 directly targeted myeloid cell leukemia-1 and activated the ATR serine/threonine kinase/checkpoint kinase 1 pathway to inhibit the proliferation, migration and invasiveness of cervical cancer cells. Li et al (27) reported that the overexpression of miR-342-3p inhibits cell proliferation, migration and invasion in cervical cell lines by targeting mammalian transcription factor forkhead box M1. Wen et al (28) reported that miR-506 induced cell cycle arrest at the G1/S transition, enhanced the apoptosis and chemosensitivity of cervical cancer cells, and inhibited cervical cancer growth in vitro and in vivo. Data from the current study provides evidence that miR-138 can inhibit proliferation, colony formation, migration and invasion, induce cell apoptosis in cervical cancer cells, and suppress tumor growth in nude mice models.

miRNAs can act as either tumor suppressors or promoters and therefore affect tumor development, proliferation, differentiation, migration and invasion (10). The expression of miR-138 is generally low in tumors, including in non-small lung cancer, colorectal cancer, neuroblastoma, esophageal squamous cell carcinoma, nasopharyngeal carcinoma and hepatocellular carcinoma (14-19). However, an association between miR-138 expression and cervical cancer has not been previously reported. To the best of our knowledge, the present study is the first to demonstrate that miR-138 expression is significantly downregulated in cervical cancer tissues and cell lines, and that low miR-138 expression is negatively associated with advanced FIGO stage and lymph node metastasis. In addition, miR-138 has been previously reported to function as a tumor suppressor in numerous malignancies by targeting various molecules (13-19). For cervical cancer, a study showed that miR-138 could significantly inhibit HeLa cell migration by targeting required for meiotic nuclear division 5 homolog A (29). However, the effect of miR-138 in cervical development and progression remains largely unknown. The present study elucidates the functionality and mechanism of the involvement of miR-138 in cervical cancer processes, and shows that miR-138 could act as tumor suppressor in cervical cancer and inhibit cervical tumor growth in vitro and in vivo by targeting hTERT.

hTERT, a catalytic subunit of telomerase, is a core component of the telomerase holoenzyme and is involved in regulating telomerase activity $(23,30)$. hTERT has been reported to be important for cancer tumorigenesis, growth, migration and 
invasion $(31,32)$. Previously, studies showed that hTERT is a target gene of miR-138 in thyroid carcinoma cell lines (33) and colorectal cancer (34). Consistent with these results, the present study identified hTERT as potential target of miR-138 in cervical cancer cells.

In conclusion, the present study has demonstrated that miR-138 expression is significantly downregulated in cervical cancer tissues and cell lines, and that decreased miR-138 expression is negatively associated with advanced FIGO stage and lymph node metastasis. miR-138 acts as a tumor suppressor in cervical cancer by suppressing cancer growth, inhibiting cell migration and invasion, and enhancing apoptosis. Furthermore, miR-138 was found to exert its function by directly targeting hTERT. These findings indicate that miR-138 may act as a novel potential therapeutic agent for the treatment of cervical cancer.

\section{References}

1. Siegel R, Naishadham D and Jemal A: Cancer statistics, 2012. CA Cancer J Clin 62: 10-29, 2012.

2. Duenas-Gonzalez A, Serrano-Olvera A, Cetina L and Coronel J: New molecular targets against cervical cancer. Int J Womens Health 6: 1023-1031, 2014.

3. de Freitas AC, Gomes Leitão Mda C and Coimbra EC: Prospects of molecularly-targeted therapies for cervical cancer treatment. Curr Drug Targets 16: 77-91, 2015.

4. Keys HM, Bundy BN, Stehman FB, Muderspach LI, Chafe WE, Suggs CL III, Walker JL and Gersell D: Cisplatin, radiation and adjuvant hysterectomy compared with radiation and adjuvant hysterectomy for bulky stage IB cervical carcinoma. N Engl J Med 340: 1154-1161, 1999.

5. Smith RA, Brooks D, Cokkinides V, Saslow D and Brawley OW: Cancer screening in the United States, 2013: A review of current American Cancer Society guidelines, current issues in cancer screening, and new guidance on cervical cancer screening and lung cancer screening. CA Cancer J Clin 63: 88-105, 2013.

6. Waggoner SE: Cervical cancer. Lancet 361: 2217-2225, 2003.

7. Cummins JM and Velculescu VE: Implications of micro-RNA profiling for cancer diagnosis. Oncogene 25: 6220-6227, 2006.

8. Miska EA: How microRNAs control cell division, differentiation and death. Curr Opin Genet Dev 15: 563-568, 2005.

9. Croce CM and Calin GA: miRNAs, cancer, and stem cell division. Cell 122: 6-7, 2005.

10. He L, Thomson JM, Hemann MT, Hernando-Monge E, Mu D, Goodson S, Powers S, Cordon-Cardo C, Lowe SW, Hannon GJ and Hammond SM: A microRNA polycistron as a potential human oncogene. Nature 435: 828-833, 2005.

11. Munker R and Calin GA: MicroRNA profiling in cancer. Clin Sci (Lond) 121: 141-158, 2011.

12. Li C, Feng Y, Coukos G and Zhang L: Therapeutic microRNA strategies in human cancer. AAPS J 11: 747-757, 2009.

13. Liang J, Zhang Y, Jiang G, Liu Z, Xiang W, Chen X, Chen Z and Zhao J: MiR-138 induces renal carcinoma cell senescence by targeting EZH2 and is downregulated in human clear cell renal cell carcinoma. Oncol Res 21: 83-91, 2013.

14. Ye XW, Yu H, Jin YK, Jing XT, Xu M, Wan ZF and Zhang XY: miR-138 inhibits proliferation by targeting 3-phosphoinositide-dependent protein kinase-1 in non-small cell lung cancer cells. Clin Respir J 9: 27-33, 2015.

15. Long L, Huang G, Zhu H, Guo Y, Liu Y and Huo J: Down-regulation of miR-138 promotes colorectal cancer metastasis via directly targeting TWIST2. J Transl Med 11: 275, 2013.
16. Chakrabarti M, Banik NL and Ray SK: miR-138 overexpression is more powerful than hTERT knockdown to potentiate apigenin for apoptosis in neuroblastoma in vitro and in vivo. Exp Cell Res 319: 1575-1585, 2013.

17. Gong H, Song L, Lin C, Liu A, Lin X, Wu J, Li M and Li J: Downregulation of miR-138 sustains NF- $\mathrm{KB}$ activation and promotes lipid raft formation in esophageal squamous cell carcinoma. Clin Cancer Res 19: 1083-1093, 2013.

18. Liu X, Lv XB, Wang XP, Sang Y, Xu S, Hu K, Wu M, Liang Y, Liu P, Tang J, et al: MiR-138 suppressed nasopharyngeal carcinoma growth and tumorigenesis by targeting the CCND1 oncogene. Cell Cycle 11: 2495-2506, 2012.

19. Wang W, Zhao LJ, Tan YX, Ren H and Qi ZT: MiR-138 induces cell cycle arrest by targeting cyclin D3 in hepatocellular carcinoma. Carcinogenesis 33: 1113-1120, 2012.

20. Solomon D, Davey D, Kurman R, Moriarty A, O'Connor D, Prey M, Raab S, Sherman M, Wilbur D, Wright T Jr and Young N; Forum Group Members; Bethesda 2001 Workshop: The 2001 Bethesda System: Terminology for reporting results of cervical cytology. JAMA 287: 2114-2119, 2002.

21. Yang H, Luo J, Liu Z, Zhou R and Luo H: MicroRNA-138 regulates DNA damage response in small cell lung cancer cells by directly rargeting H2AX. Cancer Invest 33: 126-136, 2015.

22. Livak KJ and Schmittgen TD: Analysis of relative gene expression data using real-time quantitative PCR and the 2(-Delta Delta C(T)) Method. Methods 25: 402-408, 2001.

23. Shi YA, Zhao Q, Zhang LH, Du W, Wang XY, He X, Wu S and Li YL: Knockdown of hTERT by siRNA inhibits cervical cancer cell growth in vitro and in vivo. Int $\mathrm{J}$ Oncol 45: 1216-1224, 2014.

24. Banno K, Iida M, Yanokura M, Kisu I, Iwata T, Tominaga E, Tanaka K and Aoki D: MicroRNA in cervical cancer: OncomiRs and tumor suppressor miRs in diagnosis and treatment. Scientific World Journal 2014: 178075, 2014.

25. Ribeiro J and Sousa H: MicroRNAs as biomarkers of cervical cancer development: A literature review on miR-125b and miR-34a. Mol Biol Rep 41: 1525-1531, 2014.

26. Zhou C, Li G, Zhou J, Han N, Liu Z and Yin J: miR-107 activates ATR/Chk1 pathway and suppress cervical cancer invasion by targeting MCL1. PloS One 9: e111860, 2014.

27. Li XR, Chu HJ, Lv T, Wang L, Kong SF and Dai SZ: miR-342-3p suppresses proliferation, migration and invasion by targeting FOXM1 in human cervical cancer. FEBS Lett 588: 3298-3307, 2014.

28. Wen SY, Lin Y, Yu YQ, Cao SJ, Zhang R, Yang XM, Li J, Zhang YL, Wang YH, Ma MZ, et al: miR-506 acts as a tumor suppressor by directly targeting the hedgehog pathway transcription factor Gli3 in human cervical cancer. Oncogene 34: 717-725, 2015.

29. Li J, Chen Y, Qin X, Wen J, Ding H, Xia W, Li S, Su X, Wang W, $\mathrm{Li} \mathrm{H}$, et al: MiR-138 downregulates miRNA processing in $\mathrm{HeLa}$ cells by targeting RMND5A and decreasing Exportin-5 stability. Nucleic Acids Res 42: 458-474, 2014.

30. Zhang W and Xing L: RNAi gene therapy of SiHa cells via targeting human TERT induces growth inhibition and enhances radiosensitivity. Int J Oncol 43: 1228-1234, 2013.

31. Cifuentes-Rojas C and Shippen DE: Telomerase regulation. Mutat Res 730: 20-27, 2012.

32. NoëlJF and WellingerRJ: Exposing secrets of telomere-telomerase encounters. Cell 150: 453-454, 2012.

33. Mitomo S, Maesawa C, Ogasawara S, Iwaya T, Shibazaki M, Yashima-Abo A, Kotani K, Oikawa H, Sakurai E, Izutsu N, et al: Downregulation of miR-138 is associated with overexpression of human telomerase reverse transcriptase protein in human anaplastic thyroid carcinoma cell lines. Cancer Sci 99: 280-286, 2008.

34. Qin YZ, Xie XC, Liu HZ, Lai H, Qiu H and Ge LY: Screening and preliminary validation of miRNAs with the regulation of hTERT in colorectal cancer. Oncol Rep 33: 2728-2736, 2015. 